\title{
The Small Acceptance Vertex Detector of NA61/SHINE
}

\author{
M. Deveaux ${ }^{1, \star}$, A. Aduszkiewicz ${ }^{2}, Y$. Ali $^{3}, M$. Baszczyk $^{4}$, W. Brylinski ${ }^{5}$, P. Dorosz ${ }^{4}$, S. Di Luise ${ }^{7}$,

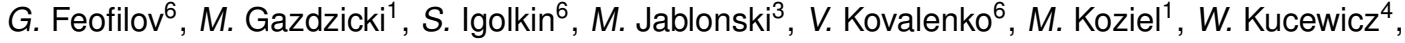

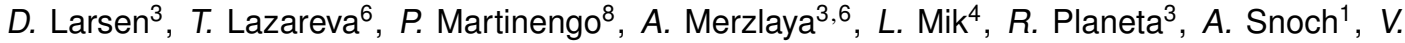 \\ Vechernin $^{6}, D$. Tefelski ${ }^{5}, M$. Suljic ${ }^{9}, P$. Staszel ${ }^{3}$, and the NA61/SHINE collaboration \\ ${ }^{1}$ Goethe University Frankfurt, Germany \\ ${ }^{2}$ University of Warsaw, Warsaw, Poland \\ ${ }^{3}$ Jagiellonian University, Cracow, Poland \\ ${ }^{4}$ AGH University of Science and Technology, Cracow, Poland \\ ${ }^{5}$ Warsaw University of Technology, Warsaw, Poland \\ ${ }^{6}$ St. Petersburg State University, St. Petersburg, Russia \\ ${ }^{7}$ Eidgenössische Technische Hochschule Zürich, Zürich, Switzerland \\ ${ }^{8}$ European Organization for Nuclear Research (CERN), Geneva, Switzerland \\ ${ }^{9}$ University \& INFN Trieste, Italy
}

\begin{abstract}
Charmonium production in heavy ion collisions is considered as an important diagnostic probe for studying the phase diagram of strongly interacting matter for potential phase transitions. The interpretation of existing data from the CERN SPS is hampered by a lack of knowledge on the properties of open charm particle production in the fireball. Moreover, open charm production in heavy ion collisions by itself is poorly understood. To overcome this obstacle, the NA61/SHINE was equipped with a Small Acceptance Vertex Detector (SAVD), which is predicted to make the experiment sensitive to open charm mesons produced in A-A collisions at the SPS top energy. This paper will introduce the concept and the hardware of the SAVD. Moreover, first running experience as obtained in a commissioning run with a $150 \mathrm{AGeV} / \mathrm{c} \mathrm{Pb}+\mathrm{Pb}$ collision system will be reported.
\end{abstract}

\section{Introduction}

The NA61/SHINE [1] experiment is a fixed target hadron spectrometer located at the CERN SPS. The experiment can be operated with hadron $(\pi, K, p)$ and heavy ion beams ( $\mathrm{Be}, \mathrm{Ar}, \mathrm{Xe}, \mathrm{Pb})$. The beam energy amounts $13-158 \mathrm{AGeV} / \mathrm{c}$ for ions and up to $400 \mathrm{GeV} / c$ for protons. Since 2009, NA61/SHINE is undertaking a systematic scan of the nuclear phase diagram, which relies on measurements with different beam energies and system sizes. This phase scan aims for obtaining an understanding of a potential first order phase transition between hadronic matter and QGP and the identification of the predicted critical endpoint of this phase transition.

$\mathrm{J} / \psi$-melting has been proposed as a suited diagnostic probe for identifying this phase transition [2] and was soon experimentally observed at the CERN-SPS. However, a precise and quantitative measurement of the yield and properties of open charm particles is required to discriminate between

\footnotetext{
^e-mail: deveaux@physik.uni.frankfurt.de
} 


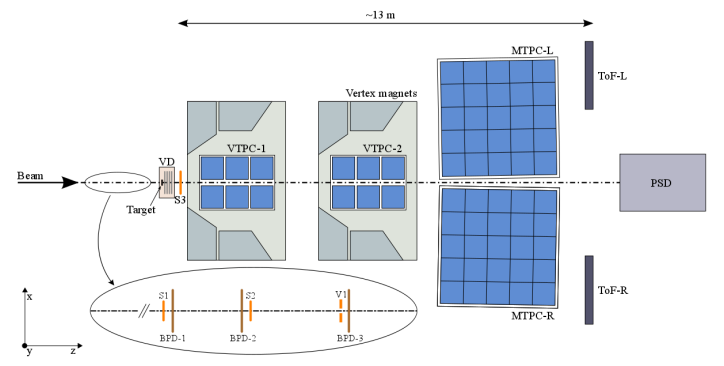

Figure 1. Sketch of the NA61/SHINE detector setup used during the $\mathrm{Pb}+\mathrm{Pb} 2016$ data taking. The position of the SAVD is shown.

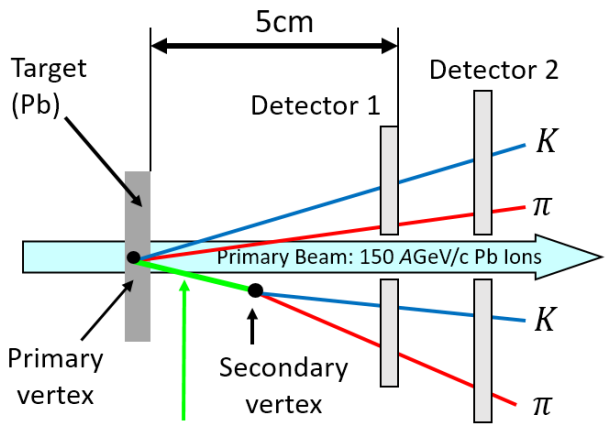

Short lived particle,

e.g. $D^{0}(c \tau=\sim 120 \mu \mathrm{m})$

Figure 2. Reconstruction strategy for open charm (not to scale). For details, see section 2.

the onset of phase transition and alternative explanations of the findings [3]. The Small Acceptance Vertex Detector (SAVD) of the NA61/SHINE experiment was build to provide the necessary data by means of doing direct measurements of open charm mesons via their hadronic decay channel (e.g. $D^{0} \rightarrow K^{-}+\pi^{+}$) at the SPS top energies.

\section{Particle reconstruction strategy and detector requirements}

Open charm mesons show a typical life-time of $c \tau \approx 100 \mu \mathrm{m}$. Their daughter particles, namely $K$ and $\pi$, are produced in large numbers by other sources in heavy ion collisions. To distinguish the daughter particles of open charm particles from those primary hadrons, one aims to accept only hadron pairs generated in a secondary decay vertex (see Figure 2). This requires to separate the latter from the primary vertex of the heavy ion collision, which comes with the need to measure the position of both vertices with excellent precision. This measurement is done by extrapolating the trajectories back to the production target and identifying intersection points. The primary vertex will typically appear as intersection point of multiple tracks while the number of tracks leaving a decay vertex remains small and well-defined.

The uncertainties of a vertex measurement are dominated by the multiple coulomb scattering of the particle in the detector material and by the limited position accuracy of the detector. To minimize both uncertainties, the detector has to provide an excellent spatial resolution. Moreover, it must be build from a minimal amount of material and located the closest possible to the primary vertex.

\section{The SAVD}

\subsection{Detector concept}

The SAVD of NA61/SHINE was designed to perform a vertex reconstruction with $\sim 70 \mu \mathrm{m}$ accuracy. It consists of two spectrometer arms composed each from four detector stations, which are located $5,10,15$ and $20 \mathrm{~cm}$ downstream the target in the stray field of the vertex magnet of NA61/SHINE (see Figure 1). The number of stations was motivated by the wish for a limited stand-alone tracking capability, which eases to match the SAVD tracks with the tracks found in the TPCs. The position of the first station forms a compromise between the need to handle the high radiation load and track 


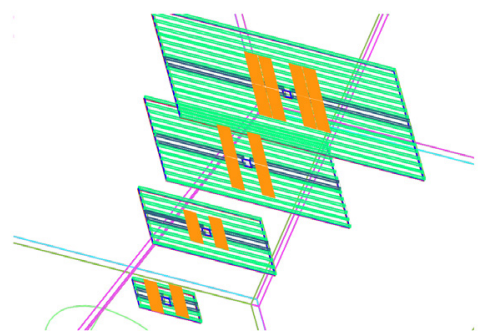

Figure 3. Active surface of the SAVD (orange) vs. the nominal geometrical acceptance of NA61/SHINE in the vertex detector region (green).

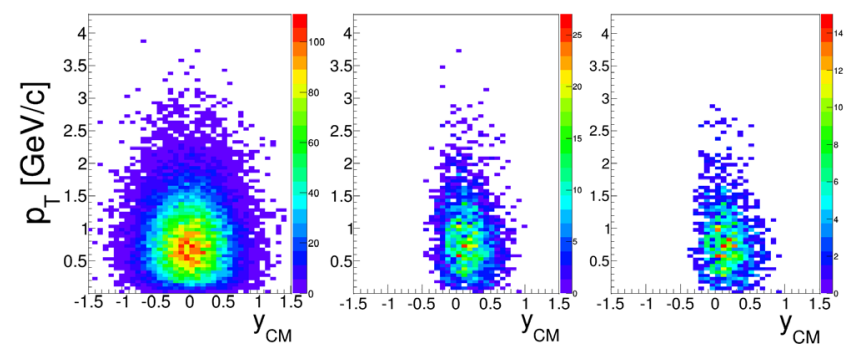

Figure 4. Simulated acceptance of the SAVD for $D^{0} \rightarrow K^{-}+\pi^{+}$ for $150 A \mathrm{GeV} / c \mathrm{~Pb}+\mathrm{Pb}$ collision systems. Left: Anticipated $4 \pi-$ emission, middle: full NA61/SHINE acceptance, right: SAVD acceptance.

density found nearby the collision point (which is eased by increasing the distance) and the need to measure the vertex position with good precision (which calls to reduce the distance).

The stations are equipped with the ultra-light, only $50 \mu \mathrm{m}$ thin, MIMOSA-26AHR CMOS sensors $[4,5]$ developed by the IPHC Strasbourg. Those silicon sensors provide a very fine pixel pitch of $18.4 \times 18.4 \mu \mathrm{m}^{2}$ resulting in an outstanding spatial resolution of $3.5 \mu \mathrm{m}$. The $1.06 \times 2.13 \mathrm{~cm}^{2}$ active surface of the sensor is covered by 1156 columns made each from 576 pixels. The readout of the pixel is done by means of a rolling shutter and the readout time is $115.2 \mu \mathrm{s}$. MIMOSA-26AHR tolerates an integrated non-ionizing dose of $>10^{13} \mathrm{n}_{\mathrm{eq}} / \mathrm{cm}^{2}$ [5] and an integrated ionizing dose of $>150 \mathrm{krad}$ [6].

The SAVD had to be realized with limited resources, which required to minimize rigorously its size and complexity. As illustrated in Figure 3, each of the first two layers of the SAVD hold only one sensor per arm. The third station and the last station hold two and four sensors per arm respectively. This turns into a total of 16 sensors with a surface of $32 \mathrm{~cm}^{2}$ and $10^{7}$ pixels, which covers only a small fraction of the geometrical acceptance of NA61/SHINE. However, thanks to the forward boost of the center-of-mass at $150 \mathrm{AGeV} / \mathrm{c}$, it is sufficient to cover mid rapidity and a substantial part of the forward rapidity (see Figure 4). Simulations [7] suggest that the SAVD accepts geometrically 35\% of all $D^{0}$ emitted into the full NA61/SHINE acceptance. During the simulations, we assumed the mean multiplicity of $D^{0}+\bar{D}^{0}$ mesons per central (20\%) $150 \mathrm{AGeV} / \mathrm{c} \mathrm{Pb}+\mathrm{Pb}$ collision to be 0.1 . Then, the number of reconstructed $D^{0}+\bar{D}^{0}$ mesons from $4 \times 10^{6}$ events amounts 1500 [8]. The S/B was estimated to amount $5(0.5)$ assuming perfect (no) particle identification respectively.

\subsection{Running conditions}

To reduce the beam-gas interactions, the SAVD is operated in a detector vessel flooded with helium, which also contains the target. The magnetic field in the detector region is not homogeneous and amounts $B<0.25 \mathrm{~T}$. The SAVD is exposed to radiation from three major sources, which are hadrons emitted from the primary collisions, delta-electrons kicked out of the target by the primary beam and direct beam ions. It was estimated that the two first radiation sources generate a radiation dose of $<10^{12} \mathrm{n}_{\mathrm{eq}} / \mathrm{cm}^{2}$ and $25 \mathrm{krad}$ during a run of 40 days. The radiation damage caused by the direct beam ions depends decisively on the beam tuning. The radiation load was estimated based on historic data from the NA61/SHINE beam counters. It was found that the most exposed point of the detector might receive $\sim 10^{9}$ ions per $\mathrm{cm}^{2}$ and run. According to the Bethe-Bloch equation, this corresponds to about $230 \mathrm{krad}$. This slightly exceeds the guaranteed capabilities of the sensor, but was considered as acceptable because only few $\mathrm{mm}^{2}$ detector surface are affected. Moreover, radiation tolerance studies 


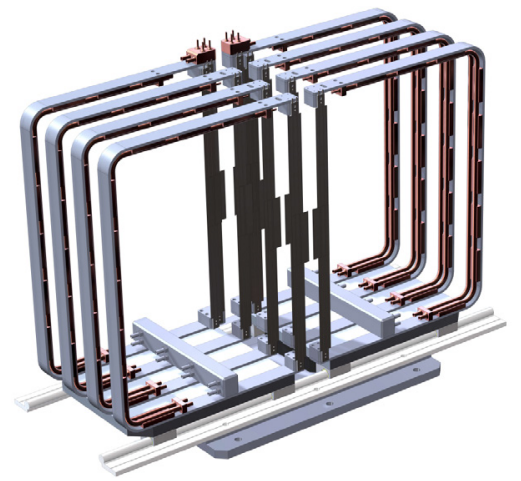

Figure 5. C-frame structure of the SAVD. The ladders, extension plates and cooling pipes are visible.

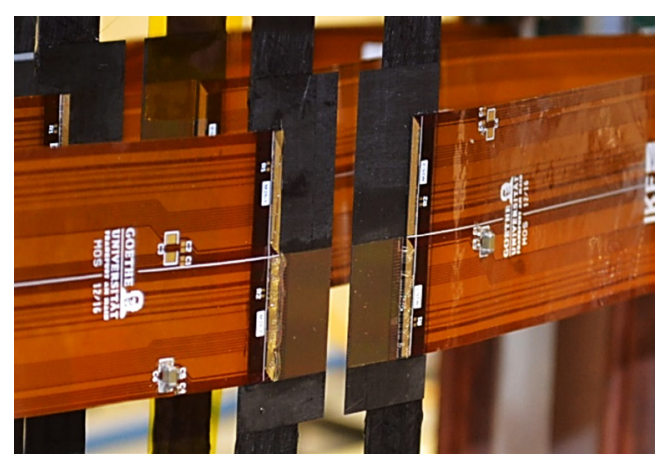

Figure 6. Photograph of the integrated detector. The sensors, extension plates, ladders and cables can be seen.

presented in [9] suggest that the reaction of MIMOSA-26AHR to modest local overdoses restricts itself likely to local losses of efficiency. The integrated non-ionizing dose was estimated to amount $4 \times 10^{11} \mathrm{n}_{\mathrm{eq}} / \mathrm{cm}^{2}$ by extrapolating theoretical results found in [10].

\subsection{Detector implementation}

The construction of the detector became possible thanks to the availability of high performance building blocks, which were kindly made available by the PICSEL group of IPHC Strasbourg and the ALICE- and CBM-collaboration. The MIMOSA-26AHR sensors were integrated on $30 \mathrm{~cm}$ long, ultra-light, actively cooled carbon fibre ladders developed for the upgrade of the ALICE Inner Barrel [11]. The width of the ladders in the sensor region was extended by means of a thin carbon fibre plate. The sensors and the dedicated, $20 \mathrm{~cm}$ long single layer flex print cables used for steering them were first glued to this plate and wedge bonded hereafter. In a next step, the integrated plate was glued to ladder. This integration step relied on tools and experiences obtained from the prototype of the CBM Micro Vertex Detector (MVD) [12].

The design of the flex print cables between the sensors and the related FEE-boards was optimized to minimize the material budget of the SAVD within the acceptance of NA61/SHINE. This was done to preserve the sensitivity of the experiment outside of the SAVD acceptance. The decision was taken knowing that, unlike to newer sensors of the MIMOSA-series, MIMOSA-26AHR is somewhat sensitive to pick-up noise injected by long cables. External filter capacitors were added to alleviate this effect. The front end boards biasing the sensors, and the TRB-data processing boards [13] providing their fast and slow control, were copied from the updated [14] CBM MVD prototype. The related firmware and software [15] was also used but had to be adapted to the needs of NA61/SHINE. Among others, the intrinsically free streaming local DAQ of the MVD prototype had to be interfaced with the triggered DAQ of NA61/SHINE.

The ladders are held by dedicated C-frames made from aluminium. During the December 2016 $\mathrm{Pb}+\mathrm{Pb}$ run reported in this work, they were complemented by one native ALICE-ITS ladder equipped with ALPIDE sensors. This was done in order to test the sensors to the high particle fluxes found in fixed target experiments. The C-frames of each arm are mounted on a magnet field tolerant moving table, which allows to move the sensors into a safe position during beam tuning or in case of a beam loss. This aims to avoid a direct impact of the beam into the sensors. Moreover, the moving engines allow to extend the only $6 \mathrm{~mm}$ wide beam gap in case necessary beam focusing is not reached. We 
found that the tables available on the market are too slow to react in a reasonable way to an unexpected beam displacement. Fortunately, we also observed that the sensors tolerate direct beam impacts without significant loss of performance (see next section). The target holder was mounted on a separate moving table.

A light and gas tight detector box was realized with metallized Plexiglas with beam entrance and exit windows made from aluminium foil. The cooling system relied on an industrial chiller using demineralized water, which was kept at modest underpressue. This underpressure and the reduced conductivity of the coolant was to reduce the consequences of a potential leakage in the cooling pipes on the nearby electronics.

\section{First operation experience}

\subsection{Immunity to heavy ions}

In 2015, the SAVD and its components were tested in beam times with a $30 \mathrm{AGeV} / \mathrm{c} \mathrm{Pb}$ beam. The first beam time focused on testing the immunity of the MIMOSA-26AHR sensors to direct beam hits. This included irradiating unpowered MIMOSA-34 test sensors with up to $1.2 \times 10^{10} 30 \mathrm{AGeV} / \mathrm{c} \mathrm{Pb}$ ions per $\mathrm{cm}^{2}$ and testing them hereafter. As discussed in more detail in [16], the test sensors survived the illumination without significant radiation damage, which is is compatible with the extrapolated predictions of [10].

Independently, an individual thick MIMOSA-26 sensor was operated directly in the heavy ion beam. This test aimed to observe the response of the sensor to heavy ions and to spot the probability of latch-ups and destructive radiation damage on the sensor. Due to missing reference detectors, the experiment was not suited to observe more subtle radiation effects like moderate losses of detection efficiency. It was found that heavy ion impacts generate clusters with about 200 fired pixels. A ring shaped after-glow is sometimes observed. As the sensor was found to be encouragingly immune to ions, it was moved to the beam spot and operated at an ion rate of about $1 \mathrm{kHz}$ (during spill) for 24 hours. We observed no hard error (e.g. stop of operation, reset, data corruption) of the sensor. Three latch-up events causing a modest over-current below the threshold of the related protection system were seen. They were recovered by manual power cycling.

\subsection{First observations from the commissioning run}

A first commissioning beam time for the SAVD with $150 \mathrm{AGeV} / \mathrm{cPb}+\mathrm{Pb}$ collision system was carried out in December 2016. During this beam time, the SAVD was operated together with the full NA61/ SHINE detector. The trigger of NA61/SHINE was tuned to record $20 \%$ central collisions, which was not optimal for open charm search but allowed for carrying out other detector tests in parallel. A total of $1.3 \times 10^{5}$ central collisions (after quality cuts, preliminary) were collected.

15 of the nominal 16 sensors were found operational and the detector operated without failure or significant damage during the full beam time. One detector arm generated about $10 \%$ of corrupted events, which was associated to a version conflict within the readout and steering software.

The data obtained from the beam test is being analysed. A track finding based on a Hough transform [17] is being used for the stand alone tracking in the SAVD. Identified tracks are extrapolated to a matching plane and merged with TPC-tracks based on position and inclination. Preliminary results show that the data obtained from the SAVD and the remaining NA61/SHINE-detector can be synchronized and that track matching is possible. After a preliminary alignment of the SAVD, a spatial resolution of $\sim 6 \mu \mathrm{m}$ (hit to track residuals) was observed. This very satisfactory result allowed to measure the primary vertex with an about $30 \mu \mathrm{m}$ precision along beam direction, which matches once more the expectations. 


\section{Summary and outlook}

The SAVD of NA61/SHINE is to reconstruct open charm particles generated in $\mathrm{Pb}+\mathrm{Pb}$ collisions at highest SPS energies. Its for stations integrate MIMOSA-26AHR CMOS sensors as provided by the IPHC Strasbourg and the ultra-light carbon fibre ladders developed for the ALICE ITS. The DAQ components and integration techniques were developed for the prototype of the CBM-detector. Despite its small active surface of $32 \mathrm{~cm}^{2}$, the SAVD covers the forward rapidity of the fireball at the nominal beam energy of $150 \mathrm{AGeV} / \mathrm{c}$. The detector was constructed, and tested among others in a beam time with $150 \mathrm{AGeV} / \mathrm{c} \mathrm{Pb}+\mathrm{Pb}$ collision system. The results confirm the feasibility of using CMOS sensor based vertex detectors in a fixed target heavy ion environment and can also be considered as a successful test of the building blocks used. Among the positive findings is the unexpectedly high immunity of the sensors to direct heavy ion hits. Moreover, a preliminary analysis of the data confirms the anticipated good spatial and vertexing resolution of the device, which provides an optimistic prospective on the success of future open charm measurements within a production run. Remaining issues like the e.g. the above mentioned software version issue are being eliminated. The improved SAVD will be used and re-tested in a Xe-La beam time end 2017 before performing a high statistics measurement in a $150 \mathrm{AGeV} \mathrm{Pb}+\mathrm{Pb}$ collision system (scheduled for end 2018). Hereafter, it is planned to decommission the SAVD and to replace it by a full-size vertex detector (Large Acceptance Vertex Detector, LAVD) during the long shut down two of the CERN SPS.

\section{Acknowledgements}

This work became possible thanks to know-how and support from the ALICE-collaboration, the CBM-collaboration, the TRB-collaboration, and the PICSEL group of IPHC Strasbourg. We wish to thank P. Klaus, J. Michel and M. Wiebusch for valuable help and training on the CBM - MVD prototype DAQ. This work was supported by the Polish National Center for Science (2014/15/B/ST2/02537). M. Deveaux is supported by HIC for FAIR.

\section{References}

[1] N. Abgrall et al. (NA61/SHINE), JINST 9, P06005 (2014)

[2] H. Satz, Nucl.Phys. B326, 613 (1989)

[3] H. Satz, Adv. High Energy Phys. 2013, 242918 (2013)

[4] A. Dorokhov et al., Nucl. Instr. and Meth. A650, 174 (2011)

[5] M. Deveaux et al., Journal of Instrumentation 6, C0 (2011)

[6] M. Koziel et al., Nucl. Instr. and Meth. A624, 437 (2010)

[7] Y. Ali, P. Staszel et al., Journal of Physics: Conference Series 509, 012083 (2014)

[8] NA61/SHINE, CERN-SPSC-2015-038 / SPSC-P-330-ADD-8 (2015)

[9] D. Deoring, Ph.D. thesis, Goethe University Frankfurt (2014)

[10] M. Xapsos et al., IEEE TNS 51, 3250 (2004)

[11] B. Abelev et al. (ALICE), J. Phys. G41, 087002 (2014)

[12] M. Koziel et al., Nucl. Instr. and Meth. 732, 515 (2013)

[13] J. Michel et al., IEEE TNS 58, 1745 (2011)

[14] P. Klaus et al., JINST 11, C03046 (2016)

[15] B. Milanovic et al., PoS TIPP2014, 404 (2014)

[16] NA61/SHINE, CERN-SPSC-2016-038 / SPSC-SR-197 (2016)

[17] A. Merzlaya et al., Journal of Physics: Conf. Series 798, 012072 (2017) 